\title{
El cuidado del familiar dependiente: análisis de género en la política social española ${ }^{1}$ The care of dependent family members: Gender analysis in Spanish social policy
}

Jesús Muyor-Rodríguez*

\section{Resumen}

Este artículo pretende contribuir al debate en torno a la producción política de los cuidados familiares en el contexto español. El objetivo de este trabajo es cuestionar la relación entre el género y los cuidados en el marco de la Ley 39/2006 de Promoción de la autonomía personal y atención a las personas en situación de dependencia. Nuestro principal interés es indagar cómo la política pública española relacionada con los servicios sociales construye y configuran todo un entramado de acción pública que transmite, refuerza y (re)produce las desigualdades de género. Utilizamos un enfoque metodológico cualitativo-interpretativo. Nos apoyamos en la revisión documental, en el análisis de discurso de normas legislativas y en la explotación de datos secundarios extraídos del Sistema público español para la Autonomía y Atención a la Dependencia.

Entre los principales resultados se destaca que la política pública española de atención a la dependencia se despliega bajo una concepción familista, responsabilizando del cuidado a las mujeres. El Sistema público de dependencia enfatiza los roles tradicionales de género y contribuye a

${ }^{1}$ Este artículo es resultado de la investigación Problematizar la diversidad funcional: sobre la (de)construcción de la dependencia y el accionar del Trabajo Social. Del contexto local a la mirada global, realizada en el Doctorado en Sociales y Jurídicas de la Universidad de JaénEspaña (2012-2016).

* Doctor por la Universidad de Jaén (España), a través del programa de doctorado en Ciencias Sociales y Jurídicas. Trabajador Social. Máster Oficial en "Dependencia e Igualdad en la Autonomía Personal" y Máster Oficial en "Gerontología Social: Longevidad, Salud y Calidad", con la especialización en "Políticas y Dirección Gerontológica", por la Universidad de Jaén (España). Profesor del Área de Trabajo Social y Servicios Sociales en la Universidad de Cádiz. Cádiz, España. Correo electrónico: jesus.muyor@uca.es.

Universidad del Valle. Cali, Colombia. Recibido: 02/05/2018 Aprobado: 23/10/2018 ISSN: 0122-1213 ISSN-e: 2389-993X Doi: 10.25100/prts.v0i27.6474 
legitimar los patrones de desigualdad existentes. Concluimos el artículo advirtiendo la necesidad de reformular la implementación de este Sistema al objeto de desresponsabilizar a las familias, especialmente a las mujeres, del cuidado. La propuesta teórica que se defiende pasa por avanzar hacia un modelo público de provisión social que sea justo y equitativo en el ámbito de la igualdad de género.

Palabras Clave: Cuidados; Dependencia; Género; Políticas sociales; Cuidadoras familiares.

\section{Abstract}

This manuscript aims to contribute to the debate around the political production of family care in the Spanish context. The objective of this work is to question the relationship between gender and care within the framework of Act 39/2006, of 14th December, on the Promotion of Personal Autonomy and Care for Dependent Persons. Our main interest is to know how Spanish public policy related to social services builds and shapes a whole network of public action that transmits, reinforces and (re) produces gender inequalities. We use a qualitative-interpretative methodological approach. We rely on the documentary review, on the discourse analysis of legislative norms and the exploitation of secondary data extracted from the Spanish Public System for Autonomy and Attention to Dependency.

Among the primary results, it is highlighted that the Spanish public policy of attention to dependency is deployed under a family concept, with responsibility for the care of women. The Public Dependency System emphasizes traditional gender roles and helps legitimize existing patterns of inequality. We conclude reporting about the need to reformulate the implementation of this System to disengage families, especially women, from care. The theoretical proposal that is defended is to move towards a public model of social provision that is fair and equitable in the field of gender equality.

Keywords: Care; Dependency; Gender; Social policies; Family caretakers.

Sumario: 1. Introducción; 2. Metodología; 3. El Estado de bienestar familista; 4. El Sistema de Autonomía y Dependencia en España: (des) responsabilizar del cuidado al familiar; 5. Entre lo cultural hecho norma, la 
necesidad económica convertida en virtud y las desigualdades perpetuadas por derecho; 6. Conclusiones; 7. Referencias bibliográficas.

\section{Introducción}

El análisis de género en las políticas familiares y de provisión del cuidado suponen un objeto de estudio central desde las ciencias sociales. La manera en la que se ha entendido y atendido las necesidades de las familias ha constituido una evidente desigualdad entre hombres y mujeres. El marco sociocultural tradicional otorga a las mujeres una función reproductora y familiar, ciñéndolas a las labores de crianza, cuidadoras y amas de casa (Cano-López, 2017).

La investigación sobre los mecanismos de gobernanza a través de las políticas de cuidados nos proporciona claves analíticas para examinar los roles y desigualdades de género. En los próximos apartados indagamos el papel que el Estado español otorga a las familias, especialmente a las mujeres, como agentes proveedores de cuidados. Nos centramos en la Ley estatal 39/2006 de Promoción de la autonomía personal y atención a las personas en situación de dependencia (LAAD). Más de diez años después de la adopción de esta relevante política pública, es imprescindible examinar de qué forma los cuidados familiares están presentes en la gobernanza de la administración pública.

\section{Metodología}

En este trabajo analizamos el papel que se le concede al cuidado familiar en la atención a la dependencia en el contexto español. Desde diferentes miradas críticas reflexionamos sobre los elementos de género y desigualdad que subyacen en los servicios sociales públicos. Utilizamos una metodología cualitativa, apoyándonos en técnicas y procesos de investigación como la revisión documental, el análisis de discurso de normas legislativas y la explotación de datos secundarios extraídos del Sistema público español para la Autonomía y Atención a la Dependencia. La metodología seleccionada nos ha permitido triangular el enfoque 
interpretativo, la investigación narrativa (Montagud-Mayor, 2015) y el conocimiento multisituado (Haraway, 1995). De esta forma incorporamos el potencial analítico del Trabajo Social por el espacio destacado que tiene entre la investigación y la práctica. Por tanto, la construcción de conocimiento se realiza también desde la propia experiencia profesional del investigador como trabajador social vinculado al Sistema español para la Autonomía y atención a la Dependencia.

\section{El Estado de Bienestar familista}

En España, al igual que en otros países de la Europa mediterránea, el pilar del bienestar se ha apoyado tradicionalmente en la familia (Esping-Andersen, 2004; Moreno, 2006). Autores clásicos como EspingAndersen (2004) consideran que el modelo de bienestar español es "extraordinariamente familista" (p. 46). Gran parte de los estudios que manifiestan el rol realizado por la familia muestran el apoyo material y afectivo de las mujeres como contribuidoras del desarrollo de políticas de bienestar (Campillo 2010; Tobío-Soler, 2013).

Resulta bastante ilustrativa la investigación realizada por Salido y Moreno (2007), para reflejar el escaso apoyo gubernamental en España desde mediados de los años 90 hasta la entrada del Sistema público de Autonomía y Atención a la Dependencia (SAAD) en el 2007. Los autores realizaron un análisis de las políticas sociales familiares de los gobiernos del Partido Popular -PP- (1996-2004) y del Partido Socialista Obrero Español -PSOE- (2004 hasta 2007). En la discusión de resultados aluden a que estos gobiernos han diseñado sus propuestas políticas considerando a las familias, muy especialmente, a las mujeres como recursos de atención y cuidado. Del intervalo que comprende desde el año 1996 al año 2004 destacan cómo las medidas de conciliación de la vida familiar y laboral se diseñan bajo la consideración de que las responsabilidades de las tareas domésticas y de cuidado familiar son propias de las mujeres y no de la sociedad en su conjunto. Estos autores reflejan que la política de mayor repercusión social propiciada por el Gobierno del PP fue la denominada como la "paga para madres trabajadoras", acometida en 2002. Se trata 
de una ayuda mensual de 100 euros para ciertas madres con niños/as menores de 3 años. Las madres deben ser trabajadoras por cuenta ajena a tiempo completo, o a tiempo parcial siempre que, como mínimo, coticen a la Seguridad Social 15 días de cada mes (jornada completa) o el 50\% de la jornada (tiempo parcial). También se incluye a las trabajadoras por cuenta propia. No obstante, estas medidas son cuestionadas por no favorecer el desarrollo de formas que superen el paradigma convencional de organización del trabajo doméstico que asume que es la mujer quien soporta la mayor carga de trabajo doméstico familiar (Salido y Moreno, 2007).

Tobío-Soler (2005) designó como la "doble presencia" al hecho por el que las mujeres participan en el mercado de trabajo pero sin abandonar la actividad doméstica. Al respecto, Durán-Heras (2005) resaltó hace más de una década que la doble presencia "es pura y simple explotación y no puede enmascararse bajo el argumento de que se trata de una opción voluntaria" (p. 3). Navarro-Ardoy (2006) centró esta cuestión a partir del análisis de género y su repercusión en la configuración de los modelos familiares. Un aspecto clave es el tipo de relación que se fragua entre la mujer y el hombre a partir del concepto de "contrato de género". Éste hace referencia a los factores culturales y las expectativas de rol que adopta cada género en distintas situaciones vitales, tanto públicas como privadas. Así, el contrato "tradicional" se materializa en el predominio de una estricta separación de roles entre mujeres y hombres, en la que a ellos les queda asignado el trabajo extra-doméstico y remunerado, mientras que a ellas se les atribuyen las tareas de cuidado del hogar y de la familia que no son remuneradas (Navarro-Ardoy, 2006).

En el ámbito de la investigación social se ha prestado especial atención a los esquemas de reparto de trabajo a partir de los análisis de los usos del tiempo. Durán-Heras y Rogero-García (2010), Rogero-García (2010a y 2010b), Meil-Landwerlin y Rogero-García (2012 y 2014), Meil-Landwerlin (2017) han abordado, en la última década, la información sobre los usos del tiempo vinculados al trabajo no remunerado (actividades domésticas y a cuidado de otras personas). La importancia de estos estudios estriba en que nos refleja modos de vida que demuestran que son finalmente las 
mujeres quienes asumen este tipo de tareas. Estas aportaciones muestran, además, cómo la falta de otros soportes públicos o privados son factores explicativos que conducen a la ausencia de las mujeres en los espacios públicos y en otros ámbitos como el del trabajo remunerado en el mercado.

Esta situación se mantiene actual a pesar de los cambios producidos en la composición familiar: familias de segundo matrimonio, las familias monoparentales, las familias que viven sin hijos, las familias reconstituidas, las familias transnacionales, etc. En cualquier caso, todas las mujeres con arreglo a elementos de género y generación comparten el hecho de que realizan grandes esfuerzos por compatibilizar el ámbito familiar y laboral (Aznar-Márquez y Belmonte-Martín, 2013). En la publicación del Instituto Nacional de Estadística (2017) se evidencia que el porcentaje de mujeres que dedican al menos varios días a la semana al cuidado de hijos y/o familiares mayores o con discapacidad o hacer tareas domésticas es superior en todos los casos al porcentaje de hombres que realizan estas mismas tareas. Con respecto al número de horas, la dedicación diaria de la mujer casi duplica la dedicación del hombre (4 horas y 29 minutos de la mujer, frente a las 2 horas 32 minutos del hombre). Respecto a las personas que realizan las actividades domésticas y de cuidado, según tipo de hogar, es superior la dedicación media diaria de las mujeres en todos los tipos de hogar, pero especialmente en el caso de familia formada por pareja con hijos (4 horas y 37 minutos la mujer, 2 horas 34 minutos el hombre). Esta diferencia de dedicación es casi la misma en el caso de pareja sola (4 horas y 45 minutos la mujer, 2 horas y 34 minutos el hombre).

Las transformaciones en los modelos de familia y sociedad no han llevado aparejado un equilibrio en las responsabilidades domésticas entre hombres y mujeres. Siguiendo con los datos publicados por el Instituto Nacional de Estadística (2017) el 91,9\% de las mujeres (de 10 y más años) dedican una media de 4 horas y 29 minutos a las tareas domésticas, de cuidado de niños, ancianos y personas dependientes. En cambio, solo el $74,7 \%$ de los hombres dedican un promedio 2 horas y 32 minutos a estas labores. Se pone de manifiesto que buena parte de los hombres no asumen las actividades relacionadas con el hogar y cuidado de familiares, y cuando las asumen hay una desigualdad en la intensidad de dedicación. Atendiendo 
a la situación laboral, las mujeres ocupadas dedican 3 horas y 46 minutos diarios a las actividades de hogar y familia y 2 horas y 21 minutos los hombres. La incorporación de las mujeres al trabajo remunerado no supone que la carga total de tareas domésticas sea menor para las mujeres, sino que se promueve la "doble jornada" o "doble presencia" de éstas en los dos ámbitos de actividad. Las responsabilidades familiares se perciben, de hecho, como un obstáculo en la trayectoria ocupacional de las mujeres. Esto se traduce en el doble esfuerzo que han de realizar las mujeres y que no se ha reducido ni en los primeros años de crisis económica española donde el desempleo en los hombres ha sido mayor que el de las mujeres (Tobío-Soler, Agulló-Tomás, Gómez, y Martín-Palomo, 2010).

La importante incorporación de la mujer al mercado de trabajo ha llevado a que la distribución de los tiempos y tareas se haya visto cuestionada con respecto a lo que sería el modelo de familia tradicional: una familia donde sólo el hombre trabaje fuera de casa y la mujer sea la que se ocupa, exclusivamente, de las tareas del hogar y del cuidado de los/as hijos y personas en situación de dependencia (Navarro-Ardoy, 2006). Examinar este hecho, junto a otras transformaciones demográficas, económicas y políticas, muestra las premisas básicas que dibujan el contexto actual en España. El aumento del envejecimiento de la población y de la esperanza de vida ha desembocado en una creciente demanda de cuidado (Abades y Rayón, 2012; Martínez-López, 2017; Rogero, 2010b). Estos hechos se han agudizado en España ya que han coincidido el envejecimiento poblacional con la expansión de las políticas neoliberales de recortes públicos, limitando severamente los recursos en materia de servicios sociales (García et al., 2017). Estas circunstancias han ayudado a conformar un panorama analíticamente complejo que vuelve a poner de manifiesto el debate público en torno a los cuidados y el tradicional "apoyo informal" que realizan las mujeres en el entorno familiar (Abellán-García y AyalaGarcía, 2012; Abellán-García, Esparza y Pérez-Díaz, 2011; Abellán et al., 2017; Durán-Heras, 2014; García-Alcaraz, Delicado-Useros, Alfaro-Espín y López-Torres, 2015). Rescatando la investigación de Salido y Moreno (2007), se seña la Ley 39/2006 de Promoción de la autonomía personal y atención a las personas en situación de dependencia (LAAD) como una de 
las políticas familiares de mayor relevancia del periodo legislativo, iniciado en 2004 y gobernado por el PSOE. Esta Ley se enmarca dentro de un nuevo Sistema para la Autonomía y Atención a la Dependencia y reconoce un nuevo derecho universal y subjetivo de ciudadanía. El propósito con el que se diseña es el de garantizar la atención y cuidados a las personas en situación de dependencia, dando así cobertura a las necesidades de los familiares y, muy especialmente, a las mujeres (Rodríguez-Cabrero, 2007).

\section{El Sistema de Autonomía y Dependencia en España: (des) responsabilizar del cuidado al familiar}

En el año 2007, con la aprobación de la LAAD, se produce una (re) configuración de los servicios sociales españoles con el propósito de implantar un sistema universal de promoción y atención de las personas en situación de dependencia (Tercera Edad y personas con discapacidad). Se dispone así, principalmente, una cobertura de servicios profesionales (domiciliarios, residenciales, centros de día y ocupacionales) y de prestaciones económicas para los cuidados familiares.

La propia LAAD en la exposición de motivos alega que:

La atención a este colectivo de población se convierte, pues, en un reto ineludible para los poderes públicos, que requiere una respuesta firme, sostenida y adaptada al actual modelo de nuestra sociedad. No hay que olvidar que, hasta ahora, han sido las familias, y en especial las mujeres, las que tradicionalmente han asumido el cuidado de las personas dependientes, constituyendo lo que ha dado en llamarse el «apoyo informal». Los cambios en el modelo de familia y la incorporación progresiva de casi tres millones de mujeres, en la última década, al mercado de trabajo introducen nuevos factores en esta situación que hacen imprescindible una revisión del sistema tradicional de atención para asegurar una adecuada capacidad de prestación de cuidados a aquellas personas que los necesitan (Ley 39 de 2006, Art. 2).

En el texto legislativo de la LAAD se justifica como línea básica de diseño de esta política pública los factores demográficos y sociales acaecidos en las últimas décadas en España. Simbólicamente, la población en situación de dependencia se presenta como un problema que tiene que 
ver sobre todo con la llamada "crisis de los cuidados" (Cerri, 2015, PérezOrozco, 2006). Se manifiesta una desestabilización del modelo tradicional de reparto de las responsabilidades sobre los cuidados, que se relaciona con una reestructuración del conjunto del sistema socioeconómico, en el que la disponibilidad de las cuidadoras familiares disminuye como consecuencia de su incorporación generalizada al empleo (Tobío-Soler, 2005). A la vez, hay una serie de cambios demográficos; la tasa de natalidad disminuye y la esperanza de vida aumenta, que hace que la demanda de atención de personas dependientes crezca en intensidad, sin que se haya alterado por ello la división sexual del trabajo en los hogares ni la segmentación de género en el mercado laboral (Ezquerra, 2012).

La LAAD podría entenderse como un impulso político para desresponsabilizar a las familias, y en especial a las mujeres, de la atención de las personas en situación de dependencia, transformando el cuidado informal en un sistema público de protección social. Fantova (2007) situaba en este punto a los servicios sociales como una pieza clave para la construcción de un nuevo pacto entre el Estado y la sociedad, para buscar nuevas sinergias entre la responsabilidad pública sobre el bienestar social y los dinamismos familiares.

En nuestras manos está, entiendo yo, aprovechar las energías positivas y oportunidades que trae esta Ley, que no son despreciables, para empujar hacia un fortalecimiento de los servicios sociales y, en particular, para la construcción, según entiendo, de un sistema público de servicios sociales cada vez más universal y equitativo, cada vez más eficaz y eficiente, amigable con las familias, atravesado por el enfoque comunitario y, con todo ello, herramienta clave para el avance de igualdad entre las mujeres y los hombres (Fantova, 2007, pp. 7).

Nos encontramos en el marco de un contexto que pretende visibilizar políticamente los discursos de género en relación a los cuidados, desplazándolos de los espacios privados y silenciados a los que tradicionalmente estaban sometidos. Una pretensión que ha servido para justificar que la LAAD en sus inicios fuera esperada y calificada como "el cuarto pilar del Estado de Bienestar" (De Lorenzo-García y 
Martínez-Rivero, 2006). El desarrollo de la Ley, no obstante, ha introducido nuevos escenarios sobre los que analizar y cuestionar las intenciones reflejadas en su redacción (Fuente de la, Y. y Sotomayor-Morales, 2015). Especialmente las relacionadas con el cuidado como responsabilidad compartida entre el Estado y la familia.

La LAAD establece la prioridad de los servicios frente a la Prestación económica para cuidados en el entorno familiar y apoyo a cuidadores no profesionales (en adelante PECEF). El propio desarrollo legislativo señala que ante un caso de una persona en situación de dependencia se debe primar que ésta reciba atención profesional de un servicio (domiciliario, residencial o centro de día) frente a una prestación económica para que sea cuidado por un familiar. En el artículo 18 de la LAAD se señala explícitamente que las PECEF son asignaciones económicas para que un familiar cuide a otro familiar dependiente, pero que están diseñadas para casos excepcionales. Concretamente, se manifiesta que podría recibirse esa prestación cuando en una zona geográfica determinada no exista cobertura de servicios profesionales y sea necesaria la atención al dependiente. Siempre y cuando el familiar cuidador pueda ejercer la actividad sin perjuicio para su bienestar y el de la persona dependiente. Sin embargo, en la implementación de la norma se evidencia que la figura del cuidado familiar tiene un gran peso desde los inicios de la puesta en marcha de la LAAD (Escudero 2007; Martín-Cano y Ruíz-Seisdedos, 2010; MartínezBuján, 2014; Minguela-Recover y Camacho-Ballesta, 2015).

Las estadísticas proporcionadas por el Sistema para la Autonomía y Atención a la Dependencia del Gobierno de España muestran que, a fecha de 31 de marzo de 2018, un 32,29\% del total de las asignaciones a beneficiarios corresponden a prestaciones para cuidados en el entorno familiar, frente a un 16,91\% que reciben ayuda a domicilio o un 13,24 que están institucionalizados en Residencias. En el sistema público español hay proporcionalmente casi las mismas personas en situación de dependencia siendo cuidadas por familiares, en su amplia mayoría mujeres de mediana edad, que recibiendo atención por el conjunto de servicios profesionales de Ayuda a domicilio, Residencias y centros de día (37,79\%). Incluso en distintos territorios geográficos los cuidados familiares superan a los 
servicios profesionales, como son la Región de Murcia, con un 53,74\%, y la Comunidad Foral de Navarra que tiene $56,93 \%$ de personas dependientes valorados por el Sistema público y cuidados por la familia (Sistema para la Autonomía y Atención a la Dependencia, 2018).

Tras más de diez años de desarrollo de la LAAD, las PECEF superan en número y proporción a cualquier otro servicio o prestación del Sistema público. Una tendencia que se ha mantenido permanentemente durante el desarrollo de la LAAD (Asociación de Directoras y Gerentes de Servicios Sociales, 2018). No obstante, los poderes públicos han ejecutado diversas actuaciones que han frenado el ritmo de crecimiento de las PECEF.

Una de las principales medidas que ralentiza la crecida de las PECEF se toma en julio de 2012 entre Comunidades Autónomas y el Gobierno Central. En el "Acuerdo del 10 de julio de 2012 del Consejo Territorial para la mejora del SAAD, publicado por la Resolución de 13 de julio de 2012 de la Secretaría de Estado de Servicios Sociales e Igualdad", se señala que;

Como medida para estimular la atención a los dependientes mediante servicios profesionalizados, generadores de empleo y actividad económica, se considera oportuno que la distribución de los créditos de los Presupuestos Generales del Estado a transferir a las Comunidades Autónomas para la financiación de la atención a la dependencia, incorpore de forma progresiva, una variable que pondere positivamente la prestación de servicios frente a la prestación económica por cuidados en el entorno familiar. La distribución entre las Comunidades Autónomas del nivel mínimo de protección, establecido en el artículo 9 de la Ley, se efectuará conforme al número de beneficiarios del Sistema y la cuantía por grado de dependencia reconocida para cada uno de ellos, ponderando positivamente la prestación de servicios respecto a la prestación económica por cuidados en el entorno familiar, en la forma que se determine reglamentariamente (Ministerio de Sanidad, Servicios Sociales e Igualdad, 2012, p. 55667).

El propio Observatorio de Dependencia de la Asociación de Directoras y Gerentes, indica que la menor asignación de PECEF "ha podido venir incentivada por un cambio en la gestión de las Comunidades Autónomas que van a resultar penalizadas en su financiación si optan por no dar servicios debido a la modificación en los criterios de distribución de 
las aportaciones estatales (Nivel Mínimo)" (Asociación de Directoras y Gerentes de Servicios Sociales, 2015, p. 24).

El Consejo Territorial del SAAD establece así una medida para incentivar económicamente a las Comunidades Autónomas que prioricen la asignación de servicios profesionales frente a las PECEF. Ante el progresivo y excesivo peso que adquieren en los primeros años de implantación de la LAAD, el SAAD se ve obligado a intentar invertir el peso, asignando más servicios profesionales que cuidados familiares. Es importante destacar que la implantación del SAAD es gradual y la entrada al sistema comenzó por las personas valoradas con gran dependencia, que es el máximo nivel reconocido. Por tanto, la mayoría de personas en situación de dependencia con necesidades de apoyo más altas estarían siendo cuidadas por familiares.

Otra de las acciones más significativas desarrolladas por el Gobierno español y que ha impactado de mayor manera en el ámbito de los cuidados familiares es el Real Decreto-Ley 20/2012, de 13 de julio, de medidas para garantizar la estabilidad presupuestaria y de fomento de la competitividad. Esta norma contiene una serie de actuaciones que, frente a la situación de crisis que atraviesa el estado español, pretenden conseguir un ahorro en el gasto público. Según indica el propio Real Decreto:

La situación económica por la que atraviesa la economía española y la exigencia de cumplimiento de los objetivos de déficit público, requieren la adopción por el Gobierno de medidas urgentes de naturaleza económica que se traduzcan en ahorros inmediatos en el gasto de las administraciones públicas (Jefatura del Estado de España, 2012, p. 50435).

En este sentido, por un lado, se reducen las cuantías máximas de las prestaciones económicas para cuidados en el entorno familiar. Por otro, las cotizaciones de las cuidadoras/es familiares en la Seguridad Social pasan a ser voluntarias y a cargo exclusivamente del familiar que ejerce el cuidado. Estos hechos implican que un/a cuidador/a familiar de una persona dependiente reconocida con el máximo grado de dependencia y, por tanto, con una intensidad de ayuda continuada durante el día para realizar las actividades básicas de la vida diaria, percibiría como máximo 442,59 euros si ha sido valorada antes del 1 de agosto de 2012, o 387,64 euros si el 
reconocimiento de dependencia es posterior. Además, si quiere cotizar a la Seguridad Social, que se consideraba obligatoria y ahora es voluntaria, tendría que abonar la/el cuidadora/or la cantidad correspondiente que antes pagaba el Estado.

Nos encontramos de esta manera, con unas políticas públicas que regulan como derecho subjetivo cuantías máximas en torno a los 400 euros para ejercer el cuidado de un familiar dependiente ${ }^{2}$. Sabiendo además que esta actividad requiere de una intensidad de atención de 24 horas al día y 354 días al año. En este sentido es importante señalar que las PECEF no pueden complementarse con ningún otro servicio profesional público. Por lo que la cuidadora familiar y la persona en situación de dependencia no tienen ningún otro servicio de cuidado público en el marco del Sistema de Autonomía y Atención a la Dependencia. De otra parte, el Estado deja de abonar la cantidad correspondiente de la Seguridad Social. Las cotizaciones tienen carácter voluntario y deberán ser pagadas por la propia persona cuidadora. No estar dada de alta en la Seguridad Social para esa actividad de cuidado familiar no se contemplaría como una situación irregular, pero no se estaría cotizando para la jubilación, incapacidad permanente, y/o prestaciones de muerte y supervivencia derivadas de accidente o de enfermedad.

A pesar del avance de estas medidas de austeridad y recortes en la prestación, como hemos mencionado con anterioridad, las cifras oficiales publicadas por el Gobierno de España señalan que la mayoría de personas en situación de dependencia están siendo atendidas por cuidadoras familiares. Lo que sí han provocado estos recortes es una disminución muy considerable de las mujeres y hombres que están dados de alta en el Régimen Especial de cuidadores no profesionales. La cifra de cuidadores/ as familiares que han dejado de cotizar en la Seguridad Social desde la implantación del Real Decreto-Ley 20/2012 se sitúa en torno un 94\% (Asociación de Directoras y Gerentes de Servicios Sociales, 2018).

Estos recortes conllevan una precarización en las condiciones en las que la unidad familiar ejerce el cuidado y un retroceso en el valor social de

${ }^{2}$ El salario medio en España es de 1.639 euros mensuales (Adecco, 2018) y el salario mínimo interprofesional es de 735,90 euros al mes (14 mensualidades) (Real Decreto 1077/2017 del Ministerio de Empleo y Seguridad Social). 
la atención a las personas en situación de dependencia. El Estado, bajo la apariencia de una prestación pública, está reduciendo su responsabilidad y transfiriendo a la familia la autogestión del cuidado, bajo peores condiciones y nuevas expresiones de desigualdad. Los cuidados familiares se utilizan como elemento de reajuste del sistema socioeconómico devolviendo la invisibilidad de este trabajo. Disminuye el peso del sector público, dando por hecho que existe un trabajo familiar para realizar lo que debe ser responsabilidad pública y para sustituir la falta de servicios básicos (PérezOrozco, 2010). En este sentido, toman fuerza de nuevo los debates desde una perspectiva feminista sobre quién realiza el cuidado (mujeres) y quiénes deberían asumirlo (cuestión de estado). Desde un punto de vista estructural se manifiesta que esta política pública no contempla una mirada de género puesto que no cuestionan elementos cruciales para el debate como la división sexual del trabajo remunerado, la redistribución de tareas en el ámbito privado o la necesidad de servicios públicos. Por el contrario, las medidas establecidas en la norma refuerzan los roles asignados por cuestión de género propios del sistema patriarcal (Martín-Cano y Ruíz-Seisdedos, 2010).

\section{Entre lo cultural hecho norma, la necesidad económica convertida en virtud y las desigualdades perpetuadas por derecho}

A pesar de que la LAAD ofrece distintas alternativas de atención, los cuidados familiares han sido, y siguen siendo, protagonistas. En las páginas anteriores hemos esbozado el proceso por el que las familias son proveedoras de servicios sociales legitimadas por la Ley. No obstante, en este análisis también conviene destacar ciertos factores socio-culturales que actúan también como mecanismos a la hora de atribuir el cuidado familiar. Cano-López (2013) introduce estos análisis desde el deber moral en el que la mayoría de las mujeres han sido socializadas. Este sociólogo señala que todavía juega un papel fundamental la construcción del valor de la buena hija, esposa o madre que se relaciona con el carácter cultural por el que se acaba asumiendo el cuidado cotidiano de un familiar:

Se trata de la reproducción social de unas prácticas incorporadas por las mujeres a través de los esquemas simbólicos de carácter cultural que 
imperan en una sociedad neoliberal regida bajo un sistema patriarcal. Las mujeres de clases populares no solo cuidan en casa porque no tienen recursos para mercantilizar el cuidado, sino porque su reputación está en juego: la construcción de "el valor de buena hija (o esposa)" depende de la consumación de la buena praxis en el cuidado cotidiano de su familiar: sus iguales juzgan, presionan y controlan -en la mayoría de las ocasiones inconscientemente, sin que exista una práctica reflexiva detrás-el trabajo de estas mujeres cuidadoras informales (Cano-López, 2013, p. 8).

El modelo de afecto maternal, el amor de madre, es el referente normativo que se manifiesta como incondicional y se traduce en el deber de una entrega absoluta de la cuidadora familiar hacia la persona dependiente (Martín-Palomo, 2014). Artiaga-Leiras (2015) refleja cómo los afectos amorosos son centrales para explicar quién realiza los cuidados, justificando la entrega y el sacrificio personal.

Esta concepción de los cuidados que favorece la exclusividad y la entrega personal sin límites en la relación afectiva inhibe la lógica de las responsabilidades sociales ante las situaciones de vulnerabilidad social, incluso en el caso de las personas que son conscientes de estas sujeciones (...). La consideración de los cuidados como una relación de amor, personal e intransferible, concibe los cuidados como un problema que ha de resolverse en el ámbito privado del hogar y no tanto como un problema social y que, como tal, requiere una respuesta colectiva (Artiaga-Leiras, 2015, p. 326).

La LAAD ha institucionalizado ese deber cultural, transfiriendo a las familias la obligación de dispensar los cuidados. Una actividad que aglutina apoyos físicos, psicológicos, sociales y de recursos económicos que son necesarios para la atención a la persona en situación de dependencia (Martín-Palomo, 2013; Martínez-Buján, 2014; Minguela-Recover y Camacho-Ballesta, 2015).

Las repercusiones del cuidado han sido abordadas desde el campo de las ciencias sociales a partir de las intensidades de apoyo (Durán-Heras, 2014). El cuidado puede implicar una dedicación exclusiva, prolongándose durante un largo tiempo, provocando que la/el cuidador/a tenga la necesidad de tener que abandonar el trabajo laboral o reducir la jornada (Rogero-García, 
2010a). Las PECEF, al ser prestaciones que tienen por finalidad garantizar los apoyos que necesitan las personas en situación de dependencia, implican una dedicación total de la cuidadora para atender las actividades básicas de la vida diaria. Este hecho supone que el/la cuidador/a se organice en función del familiar dependiente. Esto es, adaptarse a sus horarios de rutinas, de comidas, de actividades, etc., por lo que el cuidado tiene que ejercerse casi durante todo el día y, además, en otros espacios distintos a la vivienda. Pérez-Orozco (2010) se refiere a este hecho como un "círculo vicioso" que existe entre ejercer el cuidado, la generación de desigualdad y la exclusión. Esta autora señala que encargarse de los cuidados familiares impone serias limitaciones para la incorporación al mercado laboral, o el mantenimiento de jornadas prolongadas de empleo, y, por tanto, menos capacidad económica para afrontar los costes de la atención.

Es importante recordar que en los primeros años de desarrollo de la LAAD las familias españolas sufren importantes consecuencias debido a la crisis económica, especialmente por un elevado endeudamiento y una disminución, o ausencia, de ingresos (Asociación de Directoras Gerentes de Servicios Sociales, 2011; Llano, 2015; Zamora, 2014). Frente al debilitamiento de los servicios sociales (García, et al., 2017), en la elección de la estrategia de cuidado adquieren gran importancia los cálculos monetarios con el fin de buscar la máxima "rentabilidad". Martínez-Buján (2014) indica que el cuidado familiar se postula como la opción más preferible para las familias, antes que un servicio profesional público por el que no se recibe ninguna prestación económica. Este hecho, desde un punto de vista de género, puede convertir las PECEF en un mecanismo institucional que someta a muchas mujeres al ámbito familiar, reforzando el modelo que la propia LAAD pretendía minimizar.

Los hogares españoles ante la falta de ingresos económicos prefieren las PECEF como medida para paliar estas situaciones. Al tiempo que la Administración favorece esta vía, transfiriéndose a las familias la obligación de dispensar los cuidados físicos, psicológicos, sociales y de recursos económicos necesarios para atender al familiar. Los ingresos económicos condicionan las oportunidades de poder externalizar la atención mediante servicios públicos, pero también de mercantilizar el cuidado en un contexto 
en el que el Estado no asume una responsabilidad plena en la atención a la población dependiente. Las PECEF no sólo encubren que la responsabilidad sigua recayendo en los hogares sino también que las personas en situación de dependencia, que viven en unidades de convivencia con escasos recursos económicos, vean reducidas las oportunidades de satisfacer sus necesidades a través de otros apoyos complementarios al cuidado familiar. En consecuencia, el reparto de trabajos y la calidad de vida del cuidador/a y del dependiente es extremadamente desigual (Pérez-Orozco, 2014). Así, la carencia de redes sociales y los apoyos en la co-gestión de las situaciones de dependencia marcan, también, los procesos de desigualdad/exclusión de cuidadoras y dependientes. Ante la dedicación del cuidado, que incluye gran cantidad de tiempo que limita las posibilidades de construir y/o fortalecer la vida social y ante la ausencia de cambios significativos en las identidades masculinas (Colectivo loé, 2012), el cuidado sigue en manos de las mujeres. Las familias intentan desplegar diversas estrategias de conciliación, que ni son suficientes para cubrir todas las necesidades de cuidados, ni son satisfactorias para poder tener la vida deseada y, en algunos casos, se basa en una posición subordinada hacia quienes se delega el cuidado. Como señala Pérez-Orozco (2014);

Este agotarse en lo cotidiano se acompaña de delegar gran parte del cuidado a otras mujeres: abuelas, a lo largo de un eje marcado por la edad, y empleadas de hogar, a lo largo de ejes marcados por la desigualdad de clase, etnia y país que se habita y transita (p. 214).

El género continúa teniendo un papel esencial en la reorganización del cuidado de la persona dependiente. Bien dentro del propio ámbito familiar, delegando tareas a sobrinas, hijas, madres etc., o mediante la mercantilización del cuidado. En esta última opción suelen ser las mujeres inmigrantes, en ocasiones en situación administrativa irregular, las que se ocupan de la atención a las personas dependientes mediante economía sumergida, sin contrato laboral, y con retribuciones económicas más bajas que al ser contratadas por empresas (Robledo-Martín, Martín-Crespo, Pedraz-Marcos, Cabañas-Sánchez y Segond-Becerra, 2017). 


\section{Conclusiones}

En este trabajo se desvelan aspectos significativos para comprender los modos de articular la política social española en el ámbito de la atención a las situaciones de dependencia. Destacamos que la respuesta política ante la crisis económica se basa en una socialización de la deuda privada de grandes capitales (Pérez-Orozco, 2014), provocando recortes sociales que afectan directamente al conjunto de la población y, como hemos visto, de manera particular a las familias/mujeres cuidadoras. Esta disminución de la inversión en servicios sociales no solo enfatiza la autogestión de los problemas sociales, sino que plantea una cuestión central relacionada con la equidad. Esto es, la transferencia de la responsabilidad de cuidar del Estado a la familia. El Estado de Bienestar español no ha garantizado una política social que construya alternativas a las desigualdades de género. Por el contrario, la LAAD (re)produce un modelo que fuerza a las mujeres a asumir roles tradicionales y, severamente, discriminatorios.

A partir de la implementación de la LAAD, se reestructura una política tradicional familista que emerge en un nuevo contexto de crisis económica. Las familias adquieren una nueva obligación legal de responsabilizarse y ejercer el cuidado de las personas dependientes. Para algunas familias las PECEF pueden verse como un ingreso económico en momentos de dificultad, pero conlleva de forma encadenada una precarización en las formas de ejercer el cuidado y una reproducción de las desigualdades de género.

Este nuevo Sistema público de atención a la dependencia institucionaliza las discriminaciones de género. Se ha creado un modelo que perpetúa la división sexual del trabajo (ámbito privado y público) y refuerza los estereotipos asignados a los géneros. Además, el espacio privado ocupado por las mujeres cuidadoras, y otorgado por los poderes públicos, es un espacio sumamente precario. Las condiciones en las que el Estado favorece el cuidado familiar se basan en una vulneración sistemática de derechos. La cotización voluntaria a la Seguridad Social, y que debe pagar la propia cuidadora, las escasas retribuciones económicas por ejercer el cuidado y la inexistente posibilidad de apoyo público complementario conforman un 
modelo social que se cimienta sobre la invisibilización, la precarización y la vulneración de los derechos de las mujeres cuidadoras familiares.

Creemos necesario introducir estos elementos de género en el análisis de las políticas de atención a las personas en situación de dependencia. El reto que nos ocupa no es solo dar cobertura a una población mundial más envejecida sino replantearse el modelo social con el que se quiere abordar. Tenemos la responsabilidad de asumir los cuidados como una cuestión de Estado. Un desafío que nos obliga a avanzar hacia un modelo público de provisión social que sea justo y equitativo también en el ámbito de la igualdad de género.

\section{Referencias bibliográficas}

Abades, A y Rayón, E. (2012). El envejecimiento en España: ¿un reto o problema social? Gerokomos, 23(4), 151-155. doi: 10.4321/S1134-928X2012000400002. Abellán-García, A. y Ayala-García, A. (2012). Un perfil de las personas mayores en España, 2012. Indicadores estadísticos básicos. Madrid: Informes Portal Mayores.

Abellán-García, A., Esparza, C. y Pérez-Díaz, J. (2011). Evolución y estructura de la población en situación de dependencia. Cuadernos de Relaciones Laborales. 29(1), 43-67. Doi: 10.5209/rev_CRLA.2011.v29.n1.2.

Abellán, A., Pérez, J., Pujol, R., Sundstro, G., Jegermalm, M., \& Malmberg, B. (2017). Partner care, gender equality, and ageing in Spain and Sweden. International Journal of Ageing and Later Life, 11(1), 69-89. doi: 10.3384/ ijal.1652-8670.16-305.

Adecco. (2018). VI Monitor Anual Adecco sobre salarios. Primer parte. Recuperado de https://adecco.es/wp-content/uploads/2018/04/NdP-VIMonitor-Adecco-Salarios.-Parte-I.pdf.

Artiaga-Leiras, A. (2015). Producción política de los cuidados y de la dependencia: políticas públicas y experiencias de organización social de los cuidados. (Tesis Doctoral). Universidad Complutense de Madrid. Facultad de Ciencias Políticas y Sociología. Departamento de Sociología I (Cambio Social). Madrid, España. Asociación de Directoras Gerentes de Servicios Sociales. (2011). Protección de las situaciones de quiebra económica familiar desde los Servicios Sociales. Madrid: ADGSS. 
Asociación de Directoras Gerentes de Servicios Sociales (2015). XV Dictamen del Observatorio de la Ley 39/2006 de Promoción de la autonomía personal y Atención a las personas en situación de dependencia. Madrid: ADGSS.

Asociación de Directoras y Gerentes de Servicios Sociales (2018). XVIII Dictamen del Observatorio de la Ley 39/2006 de Promoción de la autonomía personal y Atención a las personas en situación de dependencia. Madrid: ADGSS.

Aznar-Márquez, J. y Belmonte-Martín, I. (2013). Las Familias como elemento subsidiario del Estado de bienestar. Revista de la Facultad de Ciencias Sociales y Jurídicas de Elche, 9, 1-20.

Cerri, C. (2015). Dependencia y autonomía: una aproximación antropológica desde el cuidado de los mayores. Athenea Digital: revista de pensamiento e investigación social, 15(2), 111-140.

Campillo, I. (2010). Políticas de conciliación de la vida laboral y familiar en los regímenes de bienestar mediterráneos: los casos de Italia y España. Política y Sociedad, 47(1),189-213.

Cano-López, T. (junio, 2013). Ley de dependencia. Un análisis crítico. En IV Congreso REPS. Las políticas sociales entre crisis y post-crisis. Red Española de Política Social (REPS). Universidad de Alcalá. Alcalá de Henares (Madrid), España. http://www3.uah.es/congresoreps2013/panel5.htm.

Cano-López, T. (2017). Tiempo y desigualdad en las dinámicas laborales y familiares. Revista Internacional de Sociología (RIS), 75(1), 1-7. doi: 10.3989/ ris.2017.75.1.16.183.

Colectivo loé. (2012). Discapacidades e Inclusión Social. Barcelona: Obra Social "La Caixa".

De Lorenzo-García, R. y Martínez-Rivero, A. (2006). La futura ley de Dependencia como pilar fundamental de la Protección Social en España. Revista española del tercer sector, 3, 49-80.

Durán-Heras, M.A. (2005). Mujeres y hombres en el siglo XXI. Cuenta y razón, (138), 45-60.

Durán-Heras, M.A. (2014). La rebelión de las familias. Mediterráneo económico, (26), 45-58.

Durán-Heras, M.A. y Rogero-García, J. (2010). La investigación sobre el uso del tiempo. Madrid: Centro de Investigaciones Sociológicas.

Escudero, B. (2007). Las políticas sociales de dependencia en España: contribuciones y consecuencias para los ancianos y sus cuidadores informales. Revista Española de Investigaciones Sociológicas (REIS), 119, 65-89.

Esping-Andersen, G. (2004). La política familiar y la nueva demografía. ICE, 81, 45-60.

Ezquerra, S. (2012). Crisis de los cuidados y crisis sistémica: la reproducción como pilar de la economía llamada real. Investigaciones Feministas, 2, 175-194. 
Fantova, F. (marzo, 2007). La Ley sobre dependencia y los servicios sociales en el País Vasco. En Forum Feminista María de Maeztu. Ley de dependencia: una perspectiva feminista. Vitoria-Gasteiz, España. Recuperado de www.fantova. net/?wpfb_dl=128.

Fuente de la, Y. y Sotomayor-Morales, E. (2015). The Spanish Long-Term Care System in the European Context. Arbor: Ciencia, pensamiento y cultura, 191(771), 1-16. doi: 10.3989/arbor.2015.771n1008

García, G., Barriga, L., Ramírez, J.M., Zubiría, A., Izquierdo, A., y Velasco, L. (2017). Índice DEC. Índice de desarrollo de los Servicios Sociales. Madrid: Asociación de Directoras Gerentes de Servicios Sociales.

García-Alcaraz, F., Delicado-Useros, MV., Alfaro-Espín, A. y López-Torres, J.D. (2015). Utilización de recursos sociosanitarios y características del cuidado informal de los pacientes inmovilizados en atención domiciliaria. Atención primaria: Publicación oficial de la Sociedad Española de Familia y Comunitaria, 47(4), 195-204.

Haraway, D.J. (1995). Ciencia, cyborgs y mujeres. La reinvención de la naturaleza. Madrid: Cátedra.

Instituto Nacional de Estadística. (2017). Mujeres y hombres en España. Madrid: Gobierno de España. Ministerio de Sanidad, Servicios Sociales e Igualdad.

Jefatura del Estado de España. (14 de diciembre de 2006). Artículo 2 [Título preliminar]. Ley de Promoción de la Autonomía y Atención a las personas en situación de dependencia. [Ley 39 de 2006]. BOE, 15.12.2006.

Jefatura del Estado de España. (13 de julio de 2012). Real Decreto-Ley de medidas para garantizar la estabilidad presupuestaria y de fomento de la competitividad. [Real Decreto-Ley 20 de 2012]. BOE-A-2012-9364.

Llano, J.C. (2015). Informe sobre el estado de la pobreza. Madrid: Red Europea de Lucha contra la Pobreza y la Exclusión Social (EAPN).

Martín-Cano, M.C. y Ruíz-Seisdedos, S. (2010). Ley de Dependencia: una mirada con perspectiva de género. Documentos de trabajo social: Revista de trabajo y acción social, 48, 84-97.

Martín-Palomo, M.T. (2013). Tres generaciones de mujeres, tres generaciones de cuidados. Apuntes sobre una etnografía moral. Cuadernos de Relaciones Laborales, 31(1), 115-138. doi: 10.5209/rev_CRLA.2013.v31.n1.41641

Martín-Palomo, M. T. (2014). Los cuidados en las familias. Un estudio a través de tres generaciones de familias en Andalucía. (Tesis doctoral). Universidad Carlos III de Madrid. Departamento de Análisis Social. Madrid, España.

Martínez-López, J.A. (2017). El modelo híbrido de atención a las personas en situación de dependencia en España: una década de cambios normativos y ajustes presupuestarios. Revista del CLAD Reforma y Democracia, 24(68), $135-168$. 
Martínez-Buján, R. (2014). Los modelos territoriales de organización social del cuidado a personas mayores en los hogares. Revista Española de Investigaciones Sociológicas (REIS), (145), 99-126. doi: 10.5477/cis/reis.145.99

Meil-Landwerlin, G.(2017). Permisos parentales para hombres y corresponsabilidad en el cuidado de niños. Revista del Ministerio de Empleo y Seguridad Social, (131), 15-34.

Meil-Landwerlin, G. y Rogero-García, J. (2012). Las Encuestas de Empleo del Tiempo como instrumento para analizar el reparto del trabajo remunerado y no remunerado. Índice. Revista de estadística y sociedad, (51), 20-22.

Meil-Landwerlin, G. y Rogero-García, J. (2014). Abuelas, abuelos y padres varones en el cuidado de la infancia. Cuadernos de Relaciones Laborales, 32(1), 49-67. doi: 10.5209/rev_CRLA.2014.v32.n1.44713

Minguela-Recover, M.A. y Camacho-Ballesta, J.A. (2015). Cuidados mixtos y cuidados informales a los mayores dependientes, ¿son complementarios o sustitutivos?: una visión comparada entre los países del sur de Europa. Zerbitzuan: Gizarte zerbitzuetarako aldizkaria = Revista de servicios sociales, (58), 15-25.

Ministerio de Sanidad, Servicios Sociales e Igualdad. (2012). Resolución de 13 de julio de 2012, de la Secretaría de Estado de Servicios Sociales e Igualdad, por la que se publica el Acuerdo del Consejo Territorial del Sistema para la Autonomía y Atención a la Dependencia para la mejora del sistema para la autonomía y atención a la dependencia. BOE, $\mathrm{N}^{\mathrm{o}} 185$.

Ministerio de Empleo y Seguridad Social. (29 de diciembre de 2017). Real Decreto por el que se fija el salario mínimo interprofesional para 2018. [Real Decreto 1077 de 2017]. BOE-A-2017-15848.

Montagud-Mayor, X. (2015). La narración de la experiencia profesional como expresión del conocimiento en Trabajo Social. Cuadernos de Trabajo Social, 28(2), 199-209. doi: 10.5209/rev_CUTS.2015.v28.n2.48971.

Moreno, L. (2006). The Model of Social Protection in Southern Europe: Enduring Characteristics? Revue Française des Affaires Socials, (1), 73-95.

Navarro-Ardoy, L. (2006). Modelos ideales de familia en la sociedad española. Revista Internacional de Sociología (RIS), 64(43), 119-138. doi: 10.3989/ ris.2006.i43.43.

Pérez-Orozco, A. (2006). Amenaza tormenta: la crisis de los cuidados y la reorganización del sistema económico. Revista de economía crítica, (5), 7-37. Pérez-Orozco, A. (2010). Cadenas globales de cuidado ¿Qué derechos para un régimen global de cuidados justo? Santo Domingo, República Dominicana: Instituto Internacional de Investigaciones y Capacitación de las Naciones Unidas para la Promoción de la Mujer (UN-INSTRAW).

Pérez-Orozco, A. (2014). Subversión feminista a la economía. Aportes para un debate sobre el conflicto capital-vida. Madrid: Traficante de sueños. 
Robledo-Martín, J., Martín-Crespo, C., Pedraz-Marcos, A., Cabañas-Sánchez, F., y Segond-Becerra, A. (2017). La externalización del cuidado de las personas mayores a mujeres migrantes: quién decide y en qué circunstancias. Pedagogía Social. Revista Interuniversitaria, (29), 183-196. doi: 10.7179/ PSRI 2017.29.13.

Rodríguez-Cabrero, G. (2007). La protección social de la dependencia en España. Un modelo sui generis de desarrollo de los derechos sociales. Política y Sociedad, 44(2), 69-85.

Rogero-García, J. (2010a). Las consecuencias del cuidado familiar sobre el cuidador: una valoración compleja y necesaria. Index de Enfermería, 19(1). 47-50.

Rogero-García, J. (2010b). Los tiempos del cuidado. El impacto de la dependencia de los mayores en la vida cotidiana de sus cuidadores. Madrid: Instituto de Mayores y Servicios Sociales (IMSERSO).

Salido, O. y Moreno, L. (2007). Bienestar y políticas familiares en España. Política y Sociedad, 44(2), 101-114.

Sistema para la Autonomía y Atención a la Dependencia. (2018). Análisis Explicativo de las Estadísticas Mensuales del Sistema para la Autonomía y Atención a la Dependencia. Situación a 31 de octubre De 2018. Instituto de Mayores y Servicios Sociales (Imserso). Recuperado de http://www. dependencia.imserso.gob.es/dependencia_01/documentacion/estadisticas/est inf/datos_estadisticos_saad/index.htm.

Tobío-Soler, C. (2005). Madres que trabajan. Dilemas y estrategias (feminismos). Madrid: Cátedra.

Tobío-Soler, C. (2013). Estado y familia en el cuidado de las personas: sustitución o complemento. Cuadernos de Relaciones Laborales, 31(1), 17-38. doi: 10.5209/rev_CRLA.2013.v31.n1.41623.

Tobío-Soler, C., Agulló-Tomás, M. S., Gómez, M.V., y Martín-Palomo, M.T. (2010). El cuidado de las personas. Un reto para el S.XXI. Barcelona: Fundación La Caixa.

Zamora, J.A. (2014). Enfrentarse a la crisis desde la perspectiva de las víctimas. En F. Lorenzo (Coord.), VII Informe sobre exclusión y desarrollo social en España. Documento de trabajo 6.2. (pp. 1-30). Madrid, España: Fundación Foessa/Cáritas.

Cómo citar:

Muyor-Rodríguez, J. (2019). El cuidado del familiar dependiente: análisis de género en la política social española. Prospectiva. Revista de Trabajo Social e intervención social, (27), 83-105. Doi: 10.25100/prts.v0i27.6474. 